\title{
An electronic tongue as a classifier tool for assessing perfume olfactory family and storage time-period
}

\author{
Amira Jarboui $^{\mathrm{a}, \mathrm{b}}$, Ítala M.G. Marx ${ }^{\mathrm{a}, \mathrm{c}}$, Ana C.A. Veloso ${ }^{\mathrm{d}, \mathrm{e}}$, Daniel Vilaça ${ }^{\mathrm{f}}$, Daniela M. Correia ${ }^{\mathrm{e}, \mathrm{f}}$, \\ Luís G. Dias ${ }^{\mathrm{a}}$, Yassin Mokkadem ${ }^{\mathrm{b}}$, António M. Peres ${ }^{\mathrm{a}, \mathrm{g}, *}$ \\ ${ }^{a}$ Centro de Investigação de Montanha (CIMO), ESA, Instituto Politécnico de Bragança, Campus Santa Apolónia, 5300-253, Bragança, Portugal \\ ${ }^{\mathrm{b}}$ Université Libre de Tunis, Avenue Khéreddine - Pacha Tunis, 30, 1002, Tunis, Tunisia \\ ${ }^{\mathrm{c}}$ LAQV/REQUIMTE, Laboratory of Bromatology and Hydrology, Faculty of Pharmacy, University of Porto, Rua de Jorge Viterbo Ferreira, 228, 4050-313, Porto, Portugal \\ ${ }^{\mathrm{d}}$ Instituto Politécnico de Coimbra, ISEC, DEQB, Rua Pedro Nunes, Quinta da Nora, 3030-199, Coimbra, Portugal \\ ${ }^{\mathrm{e}}$ CEB - Centre of Biological Engineering, University of Minho, Campus de Gualtar, 4710-057, Braga, Portugal \\ ${ }^{\mathrm{f}}$ NORTEMPRESA Perfume Lab, Rua Parque Bouça das Mouras, 56, 4715-216, Braga, Portugal \\ ${ }^{\mathrm{g}}$ Laboratory of Separation and Reaction Engineering - Laboratory of Catalysis and Materials (LSRE-LCM), ESA, Instituto Politécnico de Bragança, Campus Santa Apolónia, \\ 5300-253, Bragança, Portugal
}

\section{A R T I C L E I N F O}

\section{Keywords:}

Perfume olfactory family

Perfume storage time-period

Potentiometric electronic tongue

Linear discriminant analysis

Simulated annealing algorithm

\begin{abstract}
A B S T R A C T
The identification of more than three perfumes is difficult and no analytical tool can completely replace the human olfactory system for fragrance classification. Indeed, no analytical system can mimic the human fragrance perception, being the recognition of perfume aroma patterns by conventional or sensor-based analytical tools a challenging task. For the perfume sector, the possibility of applying fast, cost-effective and green analytical devices for perfume analysis would represent a huge economic revenue. Since the perfume aroma pattern will depend on the composition of the liquid phase and on the diffusion properties of their volatile components, this work aimed to apply a potentiometric electronic tongue, comprising non-specific cross-sensitive lipid polymeric membranes, combined with chemometric techniques, as a novel perfume classifier. The multisensors device allowed establishing perfumes' unique fingerprints, which were successfully used to discriminate men from women perfumes, to identify the perfume aroma family (Citric-Aromatic, Floral, Floral-Fruity, Floral-Oriental, Floral-Woody, Woody-Oriental and Woody-Spicy) and, assessing the perfume storage time-period ( $\leq 9$ months; 9-24 months; and, $\geq 24$ months). The established linear discriminant models were based on single-run potentiometric profiles gathered by sub-sets of sensors selected using the simulated annealing algorithm, which enabled achieving correct classification rates of $93-100 \%$ (for leave-one-out cross-validation procedure). The satisfactory performance of the electronic tongue demonstrates the versatility of the proposed approach as a practical perfume preliminary classifier sensor device, which industrial application may be foreseen in a near future, contributing to a green-sustained economic growth of the perfume industry.
\end{abstract}

\section{Introduction}

It is expected that the global market for Fragrances and Perfume exceeds US $\$ 40$ billion by 2020 [1]. A perfume may comprise from 10 to 100 individual ingredients [2], which are usually complex mixtures of synthetic or natural (e.g. essential oils) organic compounds (e.g., aldehydes, alcohols, lactones, esters and terpene derivatives). So, assessing the perfume composition, identifying the main aroma family as well as assessing perfume-stability and longevity is not a straightforward task $[1,2]$. As most of perfume ingredients are volatile or semi- volatile, gas chromatography (GC), in combination with mass spectrometry (MS) is, by far, the most used analytical technique [3]. However, GC-MS does not provide a direct qualitative information about the sensory perception of the aroma molecules, being needed to establish calibration models for correlating this qualitative information [1]. Thus, GC-Olfactometry (GCO) or GC-sniffing techniques coupled with condensed Phase Fourier-transform infrared (FTIR) spectroscopy or Time of Flight-MS (ToFMS) may be required $[2,4]$. These techniques are time-consuming, expensive and require skilled technicians, which may be beyond the economic possibilities of low-medium local perfume

\footnotetext{
* Corresponding author. Centro de Investigação de Montanha (CIMO), ESA, Instituto Politécnico de Bragança, Campus Santa Apolónia, 5300-253, Bragança, Portugal.

E-mail address: peres@ipb.pt (A.M. Peres).
} 
companies. Thus, the development of fast, low-cost and green sensorbased techniques, which may be applied on-line, to monitor in-situ perfume aroma-fragrance profiles is highly envisaged by the industry. Electronic noses (E-noses) have been proposed for perfume analysis namely for discriminating original brand perfumes or recognizing fake counterparts [5-7]; for identifying simple aromas [8-12]; for recognizing unknown fragrance mixtures [13]; to classify different perfume classes [14]; as quality control method of musk samples [15]; for generating analyte-specific fingerprints of odorants [16]; to differentiate perfumes by brand [16,17]; or, for highlight the differences of perfumes according to the producers, using odorant maps [18]. An Enose was also applied to detect counterfeit perfumed cleaner products as well as to quantify the perfume added amount [19]. Despite the satisfactory results reported so far, the identification of more than three perfumes remains difficult for the human nose and for E-nose devices with multiple sensors [14]. To overcome this problem, complex hybrid multiple statistical classifier methodologies have been proposed [14].

As an alternative/complementary approach, the present work aims, for the first time, to evaluate the possibility of using a potentiometric electronic tongue (E-tongue) together with linear discriminant analysis (LDA) coupled with the simulated annealing (SA) variable selection algorithm, as a practical perfume classifier device, minimizing or even avoiding the need of applying complex hybrid statistical techniques. Contrary to other research areas (e.g., food science [20]), in the perfume field, the use of E-tongues is not common. Only one work reported the use of a voltammetric E-tongue for perfume evaluation [21]. The study evaluated the performance of a voltammetric E-tongue to detect the type and concentration of different perfume's fragrances. On the other hand, E-tongues have been widely used to assess positive and negative sensory attributes of foods [22-28]. Moreover, sensor lipid membranes can interact with different polar compounds (e.g., phenolic compounds, esters, alcohols and aldehydes) via the establishment of electrostatic or hydrophobic interactions [29] and, since some of these chemical families are present in perfumes (as fragrances and scent ingredients), the possible application of this type of E-tongues may be foreseen. In fact, it has been reported that lipid bilayer membranes could be effectively applied within a synthetic sensing system to discriminate odorants and successfully differentiate perfumes by brand [16]. It was also shown that a simple technique like ultraviolet-visible (UV-Vis) spectrophotometry, coupled with multivariate statistical tools, allowed obtaining a preliminary chemical fingerprint of perfume samples, enabling perfume classification [30]. Therefore, and although the advantages of using an E-tongue could not be obvious, considering that perfume analysis is usually associated to the olfactory perception of aroma fragrances, its use can be foreseen. Actually, the analysis of the perfume' liquid phase, which contains the chemical compounds responsible for the aroma profile, may be extremely relevant, allowing gathering complementary but relevant chemical information of the perfumes' main fragrances notes as well as their age, i.e., the storage time-period, during which a chemical profile change is expected.

\section{Materials and methods}

\subsection{Perfume samples}

Perfume samples were supplied by NORTEMPRESA Perfume Lab (Braga, Portugal). In total, 33 independent samples were collected, being 18 women perfumes and the other 15 men perfumes, which main details are given in Table 1. According to the label information and based on the olfactory pyramid data, perfumes were grouped into 7 different main aroma/olfactory families. Women perfumes were classified as Floral ( 5 perfumes), Floral-Fruity (5 perfumes), Floral-Oriental (5 perfumes) and Floral-Woody (3 perfumes). Men perfumes were grouped into 4 aroma families, being one of them common to the women perfumes, namely Citric-Aromatic (3 perfumes), Floral-Woody (4 perfumes), Woody-Oriental (4 perfumes) and Woody-Spicy (4
Table 1

Perfume samples details (label information: sample code, type, olfactory pyramid notes, aroma family classes; and, storage time-period classes).

\begin{tabular}{|c|c|c|c|}
\hline Sample code & Type & Aroma family class & Storage time-period class \\
\hline 100001 & Woman & Floral-Fruity & 6-9 months \\
\hline 100005 & Woman & Floral & $>24$ months \\
\hline 100006 & Woman & Floral-Fruity & 9-24 months \\
\hline 100012 & Woman & Floral-Woody & $>24$ months \\
\hline 100014 & Woman & Floral-Fruity & 9-24 months \\
\hline 100015 & Woman & Floral-Oriental & $>24$ months \\
\hline 100016 & Woman & Floral-Oriental & $>24$ months \\
\hline 100017 & Woman & Floral-Woody & 9-24 months \\
\hline 100018 & Woman & Floral & 6-9 months \\
\hline 100019 & Woman & Floral-Oriental & 9-24 months \\
\hline 100020 & Woman & Floral-Woody & $>24$ months \\
\hline 100023 & Woman & Floral & $>24$ months \\
\hline 100029 & Woman & Floral-Fruity & 6-9 months \\
\hline 100031 & Woman & Floral-Oriental & 6-9 months \\
\hline 100032 & Woman & Floral-Fruity & 6-9 months \\
\hline 100033 & Woman & Floral & 6-9 months \\
\hline 100034 & Woman & Floral & 24 months \\
\hline 100040 & Woman & Floral-Oriental & 6-9 months \\
\hline 200201 & Man & Woody-Spicy & 6-9 months \\
\hline 200204 & Man & Citric-Aromatic & 6-9 months \\
\hline 200206 & Man & Woody-Oriental & 6-9 months \\
\hline 200208 & Man & Floral-Woody & $>24$ months \\
\hline 200209 & Man & Woody-Oriental & 9-24 months \\
\hline 200210 & Man & Woody-Spicy & 9-24 months \\
\hline 200216 & Man & Woody-Oriental & $>24$ months \\
\hline 200217 & Man & Woody-Oriental & $>24$ months \\
\hline 200218 & Man & Floral-Woody & $>24$ months \\
\hline 200219 & Man & Citric-Aromatic & $>24$ months \\
\hline 200221 & Man & Woody-Spicy & $>24$ months \\
\hline 200222 & Man & Woody-Spicy & 9-24 months \\
\hline 200223 & Man & Floral-Woody & $>24$ months \\
\hline 200226 & Man & Floral-Woody & 9-24 months \\
\hline 200227 & Man & Citric-Aromatic & $>24$ months \\
\hline
\end{tabular}

perfumes). The perfumes were from different production lots and had different storage time-periods (ranging from 6 to more than 24 months), being grouped into 3 main classes: 6-9 months, 9-24 months and more than 24 months. According to the label information and data from the perfume company, all perfume samples contained denatured alcohol (a mixture of ethanol with a denaturing agent) that has antimicrobial, masking and viscosity controlling functions; parfum, meaning an undisclosed mixture of several scent chemicals and ingredients used as fragrances); aqua (i.e., water); and, propylene glycol, an organic alcohol used as a skin conditioning agent, fragrance and humectant, allowing controlling the final viscosity of the perfume. Besides, the samples could contain a mixture of other ingredients, in different proportions, which could include fragrance additive and masking ingredients (e.g., hydroxyisohexyl 3-cyclohexene carboxaldehyde that has a delicate sweet, light, floral aroma; evernia prunastri that is an extract of the oakmoss; benzyl salicylate that is a salicylic acid benzyl ester; among others) and scents (e.g., limonene that has a fresh and sweet citrus aroma; coumarin that is an aromatic organic chemical compound, used as a sweet, vanilla, nutty scent; geraniol, a monoterpenoid and alcohol, which is a natural scent ingredient; butylphenyl methylpropional, an aromatic aldehyde, which is a synthetic fragrance with a strong floral scent; among others).

\subsection{UV-Vis perfume evaluation}

UV-Vis spectrophotometry was applied to acquire a preliminary insight of each perfume composition, following the experimental methodology described by Gomes et al. [30], with some adaptations. Perfume samples were firstly diluted in the proportion of 1:4000, withdrawing $2.5 \mu \mathrm{L}$ of perfume, measured using a Gilson micropipette (0.4-10 $\mu \mathrm{L})$, to a $10 \mathrm{~mL}$ glass volumetric flask, which was filled with absolute ethanol $\left(+99 \%\right.$, Extra Pure, SLR, Fisher Chemical $\left.{ }^{\circledR}\right)$. Each 


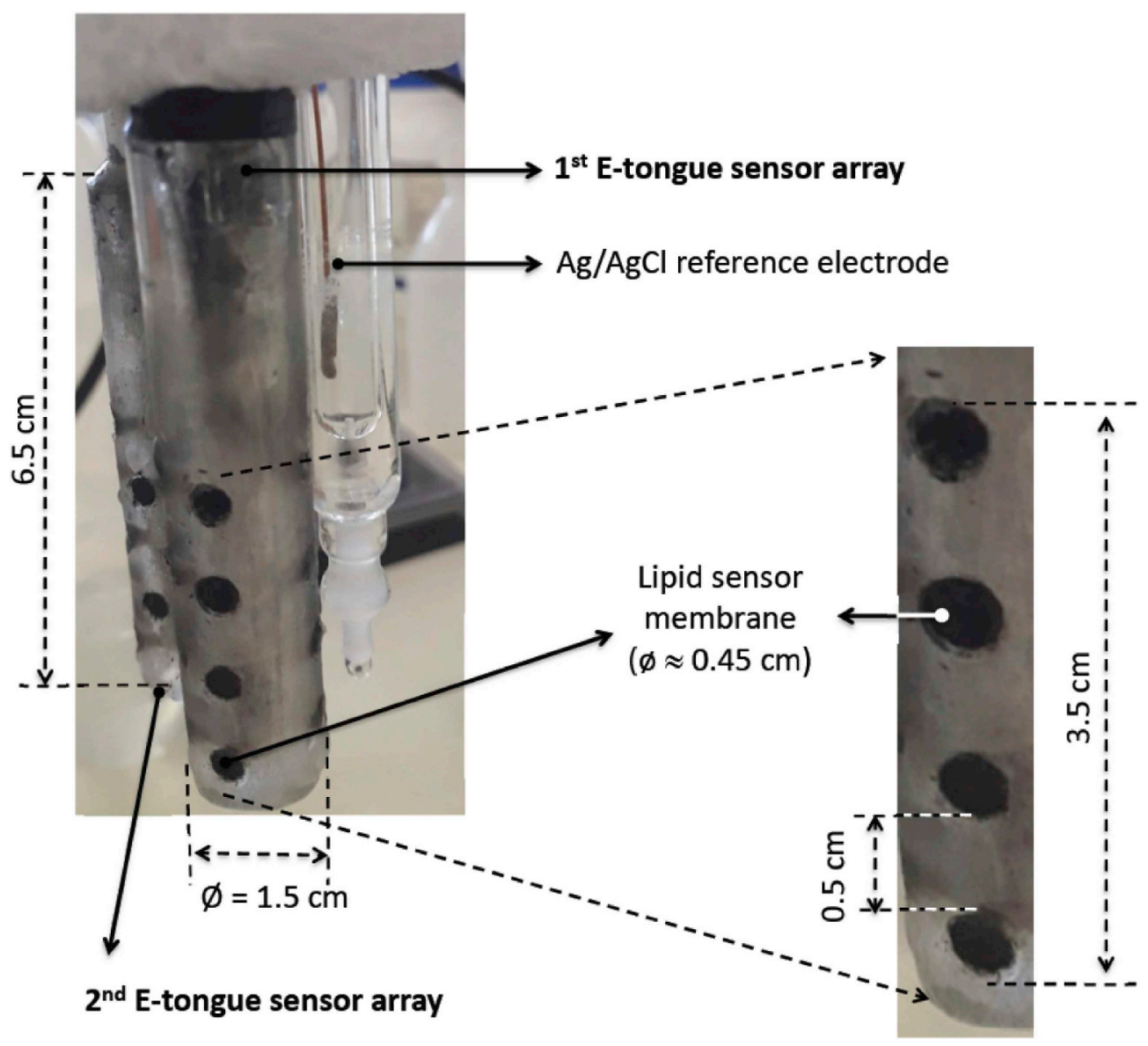

Fig. 1. E-tongue device: geometry and basic dimensions of the array and lipid membranes.

perfume-ethanol mixture was agitated, placed into a quartz cuvette (with $1 \mathrm{~cm}$ of path length) and then, the UV-Vis spectra $(200-1100 \mathrm{~nm}$, at intervals of $5 \mathrm{~nm}$ ) was recorded, using a SPECORD ${ }^{\circledR} 200$ spectrophotometer (Analytik Jena ${ }^{\circledR}$ ) and treated using the WinASPECT ${ }^{\circledR}$ software. Absorption was detected in a near UV wavelength interval (200-350 nm).

\subsection{E-tongue}

\subsubsection{E-tongue device and set-up}

A new lab-made potentiometric E-tongue multisensor device, comprising two cylindrical arrays, similar to that previous described [31], was re-designed and built (Fig. 1) specifically for the perfume analysis, aiming to minimize the total perfume volume required for each assay. Indeed, for high-value samples the required volume for each assay may be an economic concern and so, the new system was miniaturized aiming to reduce the amount of perfume used in each experimental assay. The arrays comprised the same 40 lipid polymeric cross-sensitive sensor membranes (20 sensors for each array), with the composition (lipid additive, 3\%; plasticizer, 32\%; and, polyvinyl chloride, 65\%) [31]. The sensor membranes were linked to a multiplexer Agilent Data Acquisition Switch Unit (model 34970A), which was controlled by an Agilent BenchLink Data Logger software. Each perfume analysis took $5 \mathrm{~min}$, being recorded the potentiometric signals of the 40 sensor membranes, generated by the establishment of electrostatic and/or hydrophobic interactions [29]. $\mathrm{An} \mathrm{Ag} / \mathrm{AgCl}$ double-junction glass electrode (Crison, 5241) was used as the reference electrode. The Etongue was stored in a $\mathrm{HCl}$ solution $(0.01 \mathrm{M})$ that was also used to evaluate the signals intra- and inter-day stability or the occurrence of signal drifts. Similarly, intra- and inter-day repeatability of the E-tongue potentiometric signals were also evaluated for the perfume samples of each olfactory family studied. The same sensor coding used in previous works was adopted: each sensor was identified with a letter $S$ (for sensor) followed by the number of the array ( 1 or 2 ) and the number of the membrane $(1-20$, corresponding to different combinations of plasticizers and additives).

\subsubsection{E-tongue perfume analysis: sample preparation and potentiometric assays}

Since lipid polymeric membranes were used and taking into account their possible degradation when high alcoholic solutions are being analysed, each perfume sample (that had a high level of alcohol) was previously diluted with deionized water in order to obtain an 80:20 (v/ v) water-perfume solution. This proportion was selected based on the previous experience of the research team, which observed a satisfactory E-tongue performance when used to analyse water-ethanol solutions $(80: 20, v / v)$ [31]. So, from each perfume, $8 \mathrm{~mL}$ were withdrawn and diluted in $32 \mathrm{~mL}$ of deionized water, allowing to obtain a total sample volume of $40 \mathrm{~mL}$, sufficient to completely immerse the two cylindrical E-tongue arrays, allowing the contact of the sensor membranes with the aqueous perfume solution. The solution system was then agitated during $2 \mathrm{~min}$, after which the potentiometric assays were performed in duplicate for each sample, with a third assay carried out if the recorded signals of any of the 40 sensors showed a coefficient of variation for the inter-assays greater than $20 \%$. Besides, for evaluating the sensors' intraday signal stability (i.e., signal stability over-time, for a typical daily analysis time-period), E-tongue potentiometric profiles of solutions of $\mathrm{HCl}(0.01 \mathrm{M})$ were recorded $10 \times$, in the same day, being the assays carried out over an 8-h time-period, within the usual perfume samples set of assays. 

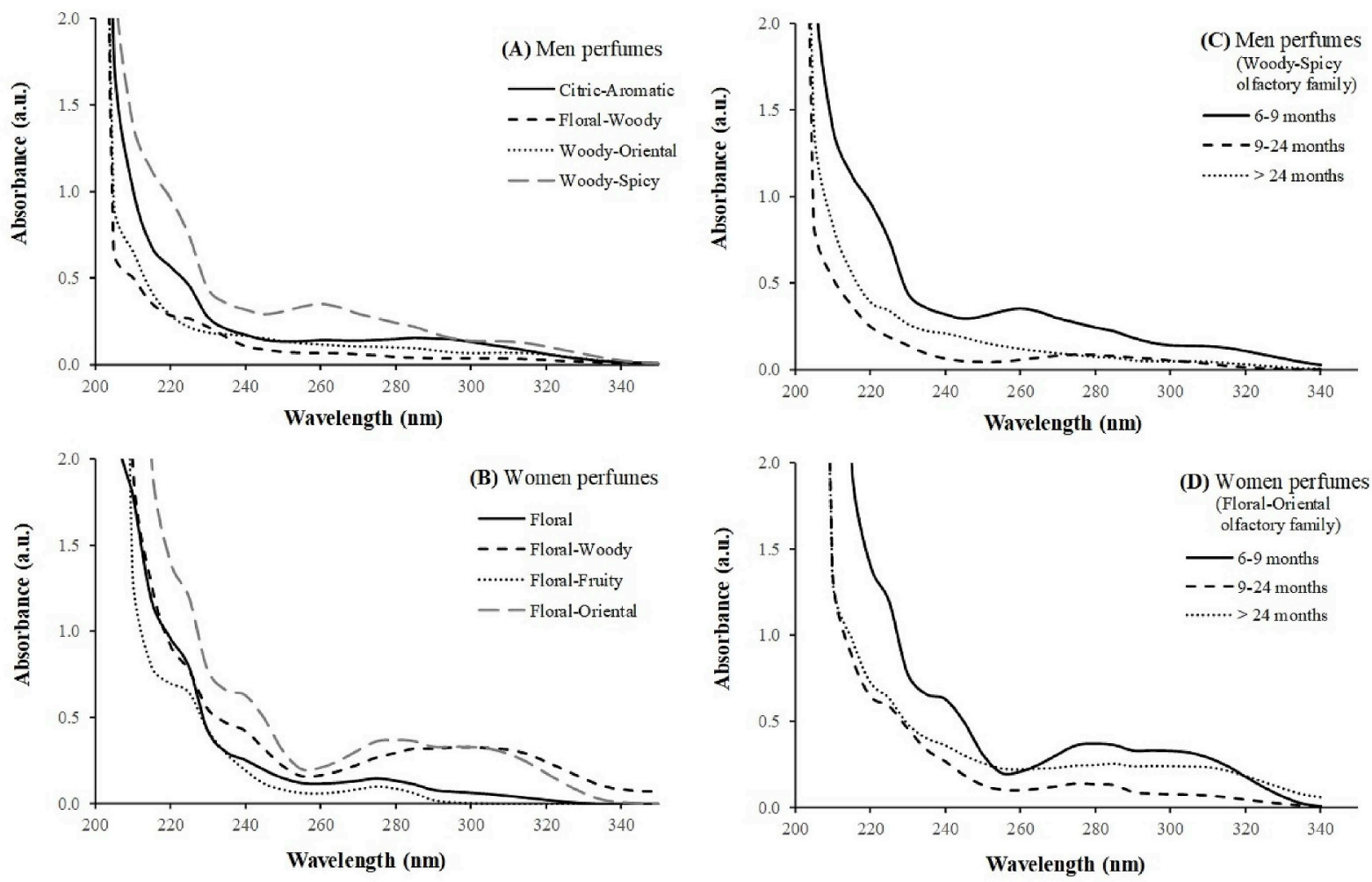

Fig. 2. UV spectra of diluted perfume samples with ethanol (1:4000 v/v) in the absorption region from 200 to $350 \mathrm{~nm}$. (A) Olfactory families of men perfumes: CitricAromatic (sample \#200204), Floral-Woody (sample \#200226), Woody-Oriental (sample \#200206) and Woody-Spicy (sample \#200201); (B) Olfactory families of women perfumes: Floral (sample \#100018), Floral-Woody (sample \#100017), Floral-Fruity (sample \#100001) and Floral-Oriental (sample \#100031); (C) Storage time-periods of Woody-Spicy men perfumes: 6-9 months (sample \#200201), 9-24 months (sample \#200210) and > 24 months (sample \#200221); (D) Storage timeperiods of Floral-Oriental women perfumes: 6-9 months (sample \#100015), 9-24 months (sample \#100031) and > 24 months (sample \# 100019).

\subsection{Statistical analysis}

Linear discriminant analysis (LDA) coupled with the meta-heuristic simulated annealing (SA) variable selection algorithm was used to evaluate the capability of the potentiometric E-tongue to differentiate men from women perfumes, to classify perfumes according to the main olfactory family and to semi-quantitatively determine the storage timeperiod. E-tongue-LDA-SA models were established based on the best sub-sets of the 40 normalized signal profiles generated during the potentiometric analysis, which were selected by the SA algorithm, aiming to minimize noise effects due to the inclusion of redundant signals. The LDA predictive performance was assessed using the leave-one-out crossvalidation (LOO-CV) technique taking into account the dimension of the independent dataset. The classification performance of each LDA model was also graphically evaluated using 2D plots of the main discriminant functions, being the class membership boundary ellipses determined based on the posterior probabilities computed using the Bayes' theorem (which enables controlling over-fitting issues) [32]. Finally, for each LDA model established the overall performance was also assessed based on the sensitivity values, i.e., based on the percentage of correct classifications, and on the specificity values, i.e., the percentage of true negatives that are correctly classified . All statistical analyses were performed using the Subselect [33,34] and MASS [35] packages of the open source statistical program R (version 2.15.1), at a $5 \%$ significance level.

\section{Results and discussion}

\subsection{UV-Vis spectra of perfume samples}

Recently, the possibility of using UV spectrophotometry in combination with chemometric techniques for perfume classification was described [30]. In the present work, it was observed that the diluted perfume-ethanol samples showed a significant absorption in the range of $200-350 \mathrm{~nm}$, corresponding to the near-UV region. Fig. 2 shows examples of the absorption spectra recorded for each olfactory family of men or women perfumes studied (Fig. 2A-B, respectively) as well as the UV spectra trend with the storage time-period for WoodySpicy men perfumes and Floral-Oriental women perfumes (Fig. 2C-D, respectively). Several peaks (major and minor bands) can be observed in the region of $210-340 \mathrm{~nm}$ that, as pointed out by Gomes et al. [30], may be due to the chemical diversity of the perfume fragrances, which include terpenoids, musks, aliphatic derivatives and aromatic derivatives, characterized by the presence of unsaturated conjugated or unconjugated carbon-carbon and/or the presence of carbonyl groups $[19,30]$. It should also be remarked that, globally, the perfume bands observed are in agreement with those found by Gomes et al. [30] for perfumes as well as for individual ethanolic standard solution of scents (e.g., limonene, linalool, citral, eugenol, coumarin, eugenol, isoeugenol and cinnamic derivatives). This similarity could be attributed to the fact that the perfumes evaluated in both studies contained several equal scents in their composition, namely, limonene, linalool, citral, coumarin, eugenol, isoeugenol, cinnamyl alcohol and cinnamal. It should also be noticed that the observed spectra confirmed the presence of polar compound families with which electrostatic and hydrophobic interactions could be established by the polymeric lipid sensor membranes comprised on the lab-made potentiometric E-tongue, as also pointed out for lipid bilayer membranes of synthetic sensing systems previously used to discriminate odorants [16]. Finally, it should be pointed out that, the UV absorption spectra recorded changed with the perfume's olfactory family and, even for the same olfactory family (e.g., Floral-Woody men and women perfumes) different absorption spectra 


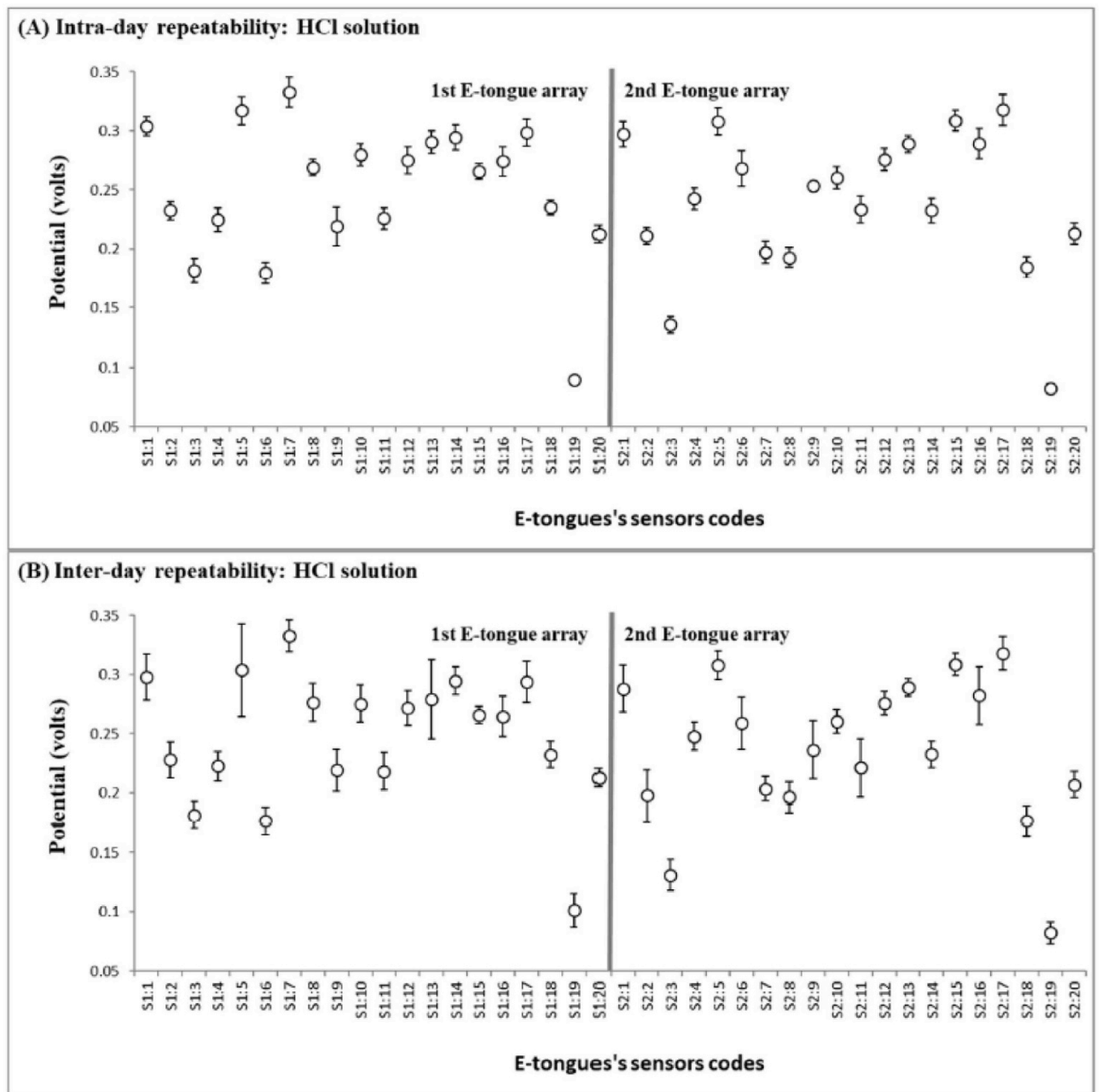

Fig. 3. E-tongue potentiometric signal profiles recorded during the analysis of a standard $\mathrm{HCl}$ solution $(0.01 \mathrm{M})$ : (A) intra-day repeatability assays (10 assays performed in the same day, within a 8-h time-period); (B) inter-day repeatability assays (12 assays performed in three consecutive days, being 4 assays carried out per day within a 8-h time-period).

were obtained (Fig. 2A-B). Indeed, it should be kept in mind that, perfumes may be classified as belonging to the same olfactory family, although having different top, heart and base olfactory notes due to the different composition in fragrances and scents. In fact, as previously stated, a perfume is a complex matrix that may comprise from 10 to 100 individual ingredients [2]. Finally, different UV absorption spectra could be observed for different storage time-periods of perfumes belonging to the olfactory family (Fig. 2C-D), being the main differences found between perfumes with less than 9 months of storage compared to those with more than 9 months of storage, showing that the perfumes, although kept in adequate storage conditions, their composition slightly change with time.

\subsection{E-tongue signal stability over time and perfume samples' signal profiles}

Potentiometric sensor devices may exhibit signal drifts, which can be minimized or overcome when daily calibrations are carried out or if signal standardization statistical treatments are applied. In which concerns potentiometric E-tongues, comprising lipid polymeric membranes (both print-screen or cylindrical arrays geometries) it was previously observed that intra-day signals were quite stable showing negligible drifts (with coefficients of variation lower than 5\%) $[22,26,28,31,36,37]$. To further check the literature reported stability of this kind of E-tongue, comprising similar sensors, $\mathrm{HCl}(0.01 \mathrm{M})$ solutions were randomly analysed $(10 \times)$, during the perfumes' assays, within the usual 8-h time-period of analysis, in one day and in three consecutive days. The results pointed out that, with the new device, the intra -and inter-day signal coefficients of variation varied, in general, in the ranges of $1.3-5.7 \%$ and $2.5-13.9 \%$, showing the overall satisfactory signal stability over-time (Fig. 3). Regarding the analysis of the diluted perfume samples (perfume-water solutions, 20:80 v/v), typical potentiometric signal profiles were acquired, varying the recorded potentials from +12 to $+340 \mathrm{mV}$, showing satisfactory intra- and interday signal repeatabilities (coefficients of variation varying from 1.8$11.4 \%$ and $4.7-16.2 \%$ ) and similar profiles to those shown in Fig. 3, although with slightly higher signal variations. The E-tongue potentiometric profiles recorded by the 40 lipid sensor membranes (1st sensor array: S1:1 to S1:20; 2nd sensor array: S2:1 to S2:20), showed slightly differences (regarding signal intensity/signal dynamic range) according to the perfume olfactory family (7 different olfactory families; men perfumes: Citric-Aromatic, Floral-Woody, Woody-Oriental and Woody-Spicy; women perfumes: Floral, Floral-Fruity, Floral-Oriental and Floral-Woody).

\subsection{E-tongue classification performance}

The performance of the proposed potentiometric E-tongue, comprising non-specific and cross-sensitive sensors, for simultaneously classifying, based on a single-run assay, the perfume type (men or women), perfume main aroma/olfactory family and perfume storage time-period was evaluated for the first time. This type of sensor device has been reported as a powerful taste sensor device for assessing 
different positive and negative sensory attributes of foods [22-28]. Moreover, the use of a multisensors arrays, with the above-mentioned characteristics may allow gathering the unique fingerprint of a perfume and so, overcoming the known limitation of applying a single sensor, which results in and unspecific response towards the complex perfume composition (10-100 individual ingredients [2]) that can deliver exactly the same potentiometric signal for different chemical compounds in solution, which are related to the specific aroma/olfactory perfume notes [3].

\subsubsection{Discrimination of men and women perfumes}

Although men and women perfumes may be differentiated according to the olfactory notes, for the perfume industry it is important to have an analytical technique that could be implemented (on-line and in-situ) for monitoring the production line, allowing a fast and easy discrimination of men from women perfumes. So, the E-tongue performance was evaluated keeping in mind this objective. An E-tongueLDA-SA model was established based on the potentiometric data of 12 sensors (1st array: S1:3, S1:4; S1:7, S1:14 and S1:20; 2nd array: S2:2, S2:4, S2:6, S2:9, S2:12, S2:13 and S2:17), enabling to correctly differentiate men from women perfumes, with sensitivities and specificities (overall and for each group) of $100 \%$ for both original grouped data (Fig. 4) and LOO-CV internal-validation procedure. All samples were correctly classified, pointing out the versatility and powerful of the classifier potentiometric device for discriminating men and women perfumes, comprising a total of 7 different olfactory families and have being stored during 6 to more than 24 months. The satisfactory results also strengthen the initial idea that E-tongues could be a practical tool for perfume analysis even if, at a first view it was expected to correlate a sensor-based device with the olfactory profile of a perfume sample.

\subsubsection{Classification of perfumes according to the main aroma family}

Men and women perfumes possess a complex composition, being a mixture of a multitude of ingredients, which include a basis of alcohol

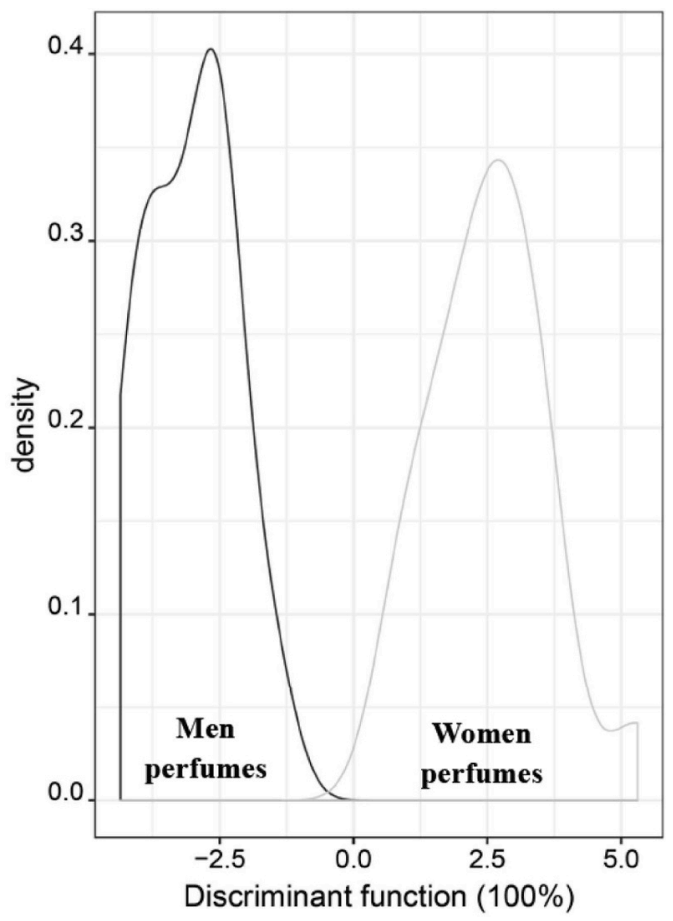

Fig. 4. Density distribution (one-dimension plot) for the discriminant function of the E-tongue-LDA-SA classification model based on 12 selected sensors' signals (1st array: S1:3, S1:4; S1:7, S1:14 and S1:20; 2nd array: S2:2, S2:4, S2:6, S2:9, S2:12, S2:13 and S2:17) established for discriminating men and women perfumes, regardless the perfume's olfactory family or storage time-period. denatured, parfum, aqua and propylene glycol combined with several other chemical compounds (e.g., fragrances and scents). Depending of the different top, heart and base olfactory notes (olfactory pyramid), each perfume may be commercially classified according to the main aroma/olfactory family (Table 1). Thus, in this study it was evaluated the E-tongue performance for classifying perfumes taking into account the main olfactory family, independently of the perfume type (men or women) or the perfume's storage time-period (i.e., perfume's age), using a LDA-SA chemometric approach. The 33 perfumes were grouped into 7 different olfactory families (Table 1 ) including, Citric-Aromatic (3 men perfumes with 6-9 months or more than 24 months of storage), Floral (5 women perfumes with 6-9 months or more than 24 months of storage), Floral-Fruity (5 women perfumes with 6-9 months or 9-24 months of storage), Floral-Oriental (5 women perfumes with 6-9 months, 9-24 months or more than 24 months of storage), FloralWoody (4 men and 3 women perfumes with 9-24 months or more than 24 months of storage), Woody-Oriental (4 men perfumes with 6-9 months, 9-24 months or more than 24 months of storage) and WoodySpicy (4 men perfumes with 6-9 months, 9-24 months or more than 24 months of storage). For this purpose, a classification E-tongue-LDA-SA model, which 2 first discriminant functions accounted for $99.87 \%$ of the total variance, was developed based on the potentiometric signals gathered by 18 selected sensors (1st array: S1:1, S1:2, S1:4, S2:6, S1:7, S1:8, S1:11, S1:12 and S1:16; 2nd array: S2:1, S2:2, S2:11 and S2:13 to S2:18). The model allowed obtaining overall sensitivities (i.e., percentage of correct classifications) of $100 \%$ and $94 \%$ and global specificities (i.e., the proportion of true negatives that are correctly classified) of $100 \%$ and $95 \%$ for the original grouped data (Fig. 5) and for the LOO$\mathrm{CV}$ internal-validation procedure, respectively. An overall satisfactory predictive performance was achieved, being the olfactory family of only 2 of the 33 perfumes incorrectly assessed (Table 2), being both predictive sensitivity and specificity per group (LOO-CV procedure) within

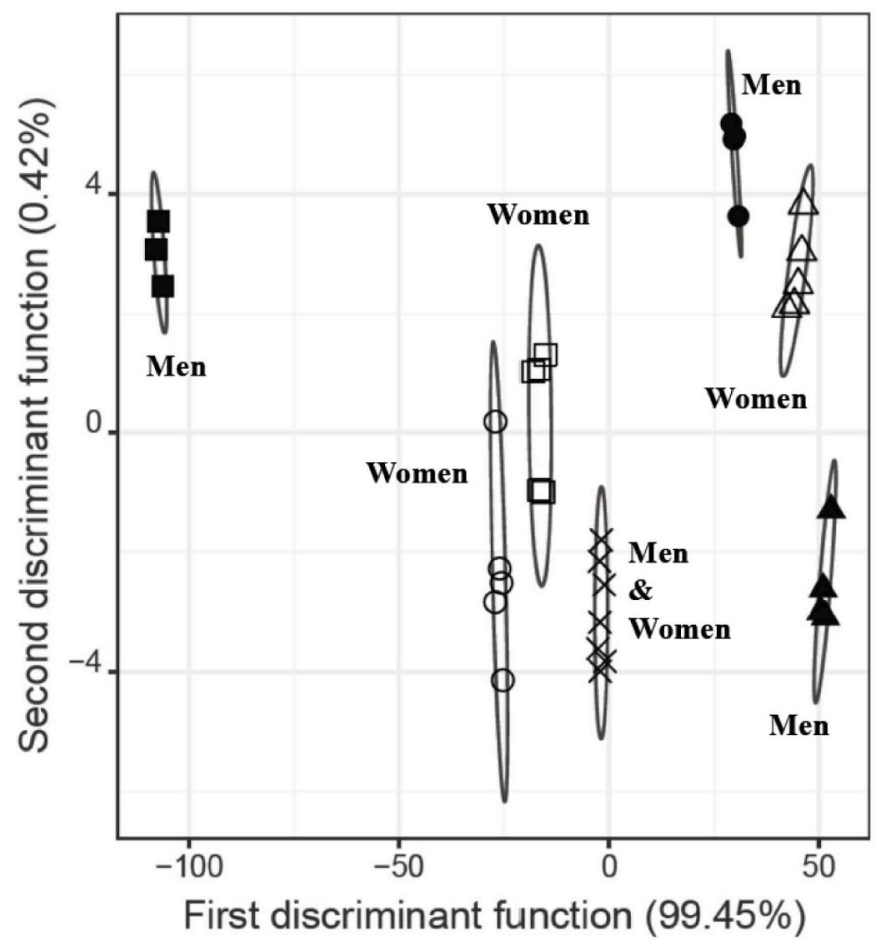

Fig. 5. Perfumes' discrimination (2D plot of the first 2 discriminant functions and respective class membership boundary ellipses) according to the main aroma/olfactory family ( $\square$ Citric-Aromatic, $\bigcirc$ Floral, $\Delta$ Floral-Fruity, $\square$ FloralOriental, $\times$ Floral-Woody, Woody-Oriental and $\boldsymbol{\Delta}$ Woody-Spicy; being fill symbols used for men fragrances, open symbols for women fragrances and other symbols for men \& women fragrances), regardless the perfume type (men or women) and the storage time-period. 
Table 2

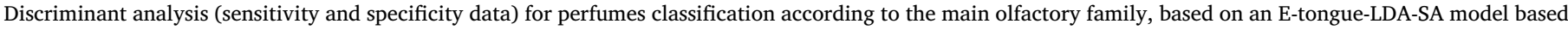

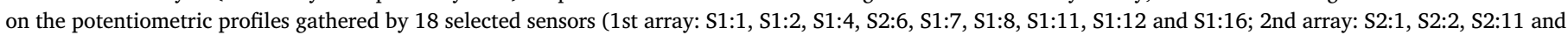
S2:13 to $S 2: 18)$.

\begin{tabular}{|c|c|c|c|c|c|c|c|c|c|}
\hline \multirow[t]{2}{*}{ Actual perfume olfactory family } & \multicolumn{7}{|c|}{ Predicted perfume olfactory family (LOO-CV internal validation procedure) } & \multirow[t]{2}{*}{ Total } & \multirow[t]{2}{*}{ Sensitivity (\%) } \\
\hline & Citric-Aromatic & Floral & Floral-Fruity & Floral-Oriental & Floral-Woody & Woody-Oriental & Woody-Spicy & & \\
\hline Citric-Aromatic & 3 & 0 & 0 & 0 & 0 & 0 & 0 & 3 & 100 \\
\hline Floral & 0 & 5 & 0 & 0 & 0 & 0 & 0 & 5 & 100 \\
\hline Floral-Fruity & 0 & 0 & 4 & 0 & 0 & 0 & 1 & 5 & 80 \\
\hline Floral-Oriental & 0 & 0 & 0 & 5 & 0 & 0 & 0 & 5 & 100 \\
\hline Floral-Woody & 0 & 0 & 0 & 1 & 6 & 0 & 0 & 7 & 86 \\
\hline Woody-Oriental & 0 & 0 & 0 & 0 & 0 & 4 & 0 & 4 & 100 \\
\hline Woody-Spicy & 0 & 0 & 0 & 0 & 0 & 0 & 4 & 4 & 100 \\
\hline Total & 3 & 5 & 5 & 6 & 6 & 4 & 5 & 33 & 94 \\
\hline Specificity(\%) & 100 & 100 & 100 & 83 & 100 & 100 & 80 & 95 & \\
\hline
\end{tabular}

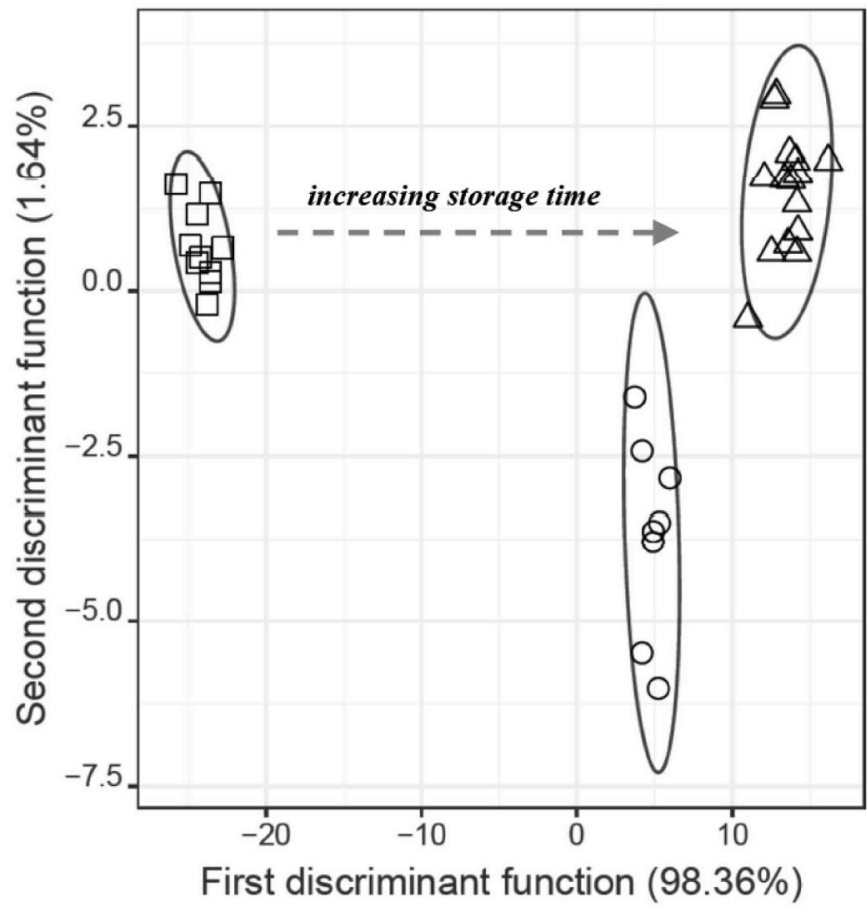

Fig. 6. Perfumes' storage time-period ( $\square$ 6-9 months; $\bigcirc 9-24$ months; $\Delta>24$ months) assessment (2D plot and respective class membership boundary ellipses) using an E-tongue-LDA-SA classification model based on the potentiometric signals of 20 selected lipid sensor membranes (1st array: S1:3, S1:4, S2:6, S1:7, S1:9, S1:12, S1:14, S1:15, S1:19 and S1:20; 2nd array: S2:2 to S2:6, S2:9, $S 2: 12, S 2: 14, S 2: 18$ and $S 2: 20$ ), regardless the type of perfume (men or women) and the aroma/olfactory family.

the range of $80-100 \%$. It should be noticed that, if the perfumes were split by men or women type, $100 \%$ of correct predictive classifications could be obtained (LOO-CV procedure) using LDA models based on the signal profiles of 8 and $9 \mathrm{E}$-tongue sensors, respectively, selected by the SA algorithm (data not shown). The overall correct classification rates achieved with the lab-made potentiometric E-tongue are of the same order of magnitude as those reported in the literature using E-nose devices coupled with different chemometric techniques (which predictive sensitivities ranged from 71 to $98 \%$ when classifying different perfume classes or discriminating them by brand) $[14,16]$ or even with a voltammetric E-tongue [21]. Furthermore, compared to the reported performances achieved with E-nose These results showed, for the first time, that a potentiometric E-tongue could be used as a classifier sensor device for perfume analysis, namely for identifying the main olfactory family. This is of utmost practical and economical relevance since this evaluation and classification requires the availability of trained sensory
Table 3

Discriminant analysis (sensitivity and specificity data) for perfumes classification according to the storage time-period, based on an E-tongue-LDA-SA model based on the potentiometric profiles gathered by 20 selected sensors (1st array: $\mathrm{S} 1: 3, \mathrm{~S} 1: 4, \mathrm{~S} 2: 6, \mathrm{~S} 1: 7, \mathrm{~S} 1: 9, \mathrm{~S} 1: 12, \mathrm{~S} 1: 14, \mathrm{~S} 1: 15, \mathrm{~S} 1: 19$ and S1:20; 2nd array: $\mathrm{S} 2: 2$ to S2:6, S2:9, S2:12, S2:14, S2:18 and S2:20).

\begin{tabular}{|c|c|c|c|c|c|}
\hline \multirow[t]{2}{*}{$\begin{array}{l}\text { Actual perfume } \\
\text { storage time- } \\
\text { period }\end{array}$} & \multicolumn{3}{|c|}{$\begin{array}{l}\text { Predicted perfume storage time-period } \\
\text { (LOO-CV internal validation } \\
\text { procedure) }\end{array}$} & \multirow[t]{2}{*}{ Total } & \multirow[t]{2}{*}{ Sensitivity (\%) } \\
\hline & $\begin{array}{l}6-9 \\
\text { months }\end{array}$ & $\begin{array}{l}\text { 9-24 } \\
\text { months }\end{array}$ & $\begin{array}{l}>24 \\
\text { months }\end{array}$ & & \\
\hline 6-9 months & 10 & 0 & 0 & 10 & 100 \\
\hline 9-24 months & 0 & 7 & 1 & 8 & 88 \\
\hline$>24$ months & 0 & 0 & 15 & 15 & 100 \\
\hline Total & 10 & 7 & 16 & 33 & 97 \\
\hline Specificity(\%) & 100 & 100 & 94 & 98 & \\
\hline
\end{tabular}

panelists, leading to an expensive and time-consuming task that may be beyond the economic possibilities of local small-medium perfume companies.

\subsubsection{Assessment of the storage time-period of the perfume samples}

For the perfume industry it is relevant to have a fast and userfriendly analytical tool for classifying perfumes according to the storage time-period (i.e., the time after production until commercialization). This possibility is even of greater practical application if it could be used regardless the type of perfume (men or women) and the perfume's aroma/olfactory family. So, the E-tongue performance to assess the storage time-period (6-9 months; 9-24 months; and more than 24 months) was further evaluated. An E-tongue-LDA-SA model, with two discriminant functions (accounting $98.36 \%$ and $1.64 \%$ of the total variability, respectively), was established based on the potentiometric signals recorded by a sub-set of 20 sensors selected by the SA algorithm (1st array: S1:3, S1:4, S2:6, S1:7, S1:9, S1:12, S1:14, S1:15, S1:19 and S1:20; 2nd array: S2:2 to S2:6, S2:9, S2:12, S2:14, S2:18 and S2:20). The multivariate linear classification model allowed an overall correct classification of the storage time-period of $100 \%$ of the original data samples (Fig. 6) and of $97 \%$ of the samples for the LOO-CV internal validation procedure (being only one sample of the 9-24 months erroneously classified as being stored for more than 24 months). The model overall specificities were of $100 \%$ and $98 \%$ for the original grouped data and for the LOO-CV procedure, respectively. The sensitivity and specificity per group, for LOO-CV procedure, ranged from 88$100 \%$ and $93-100 \%$, respectively, as shown in Table 3 . The predictive performance achieved was very satisfactory considering the variability of the perfumes included in each storage time-period (6-9 months: 3 men and 7 women perfumes from Citric-Aromatic, Floral, Floral-Fruity, Floral-Oriental, Woody-Oriental and Woody-Spicy olfactory families; 
9-24 months: 4 men and 4 women perfumes from Floral-Fruity, FloralOriental, Floral-Woody, Woody-Oriental and Woody-Spicy olfactory families; and, $>24$ months: 8 men and 7 women perfumes from CitricAromatic, Floral, Floral-Oriental, Floral-Woody, Woody-Oriental and Woody-Spicy olfactory families). This fact, clearly pointed out the versatility of the E-tongue-LDA-SA proposed approach, which has proven to be a powerful semi-quantitative classifier tool of perfume's age assessment. Furthermore, if the perfumes were split by men and women type, the correct predictive classification percentages (sensitivity values) would reach $100 \%$ (E-tongue-LDA-SA models based on the signal profiles of 7 selected sensors; data not shown), strengthen the above-mentioned powerful of the classifier potentiometric device.

\section{Conclusions}

The present study outlined, for the first time, the application of an E-tongue for perfume analysis, which allowed, in a single-run assay, to establish a unique perfume potentiometric fingerprint capable of discriminating men and women perfumes, for differentiating perfumes according to the main olfactory family and for semi-quantitatively assessing the storage time-period of perfumes. The work also highlighted the predictive satisfactory performance of a multisensor device, comprising non-specific lipid polymeric membranes, coupled with classification chemometric techniques and variable selection algorithm, showing that the proposed approach could be used by the perfume industrials as a practical, cost-effective and fast perfume classifier analytical technique as well as a complementary sensory preliminary tool, minimizing the need to recourse to trained/official perfume panelists. Thus, the study carried out may also contribute to enlarge the Etongue field of application, mainly focused on the food and environmental analysis, to the perfume emerging and promising area. Several challenging applications may be foreseen in the future for electrochemical based sensor devices, namely to monitor the maceration and maturation critical phases of a perfume design, to detect the presence of legally restricted or forbidden fragrance-related substances or even to recognize the perfume brand allowing discriminating original and copied perfumes.

\section{Conflict of interest}

The authors declare that they have no conflict of interest.

\section{Ethical approval}

This article does not contain any studies with human participants or animals performed by any of the authors.

\section{Informed consent}

Not applicable.

\section{Acknowledgments}

This work was funded by Associate Laboratory LSRE-LCM - UID/ EQU/50020/2019, strategic funding UID/BIO/04469/2019 - CEB and BioTecNorte operation (NORTE-01-0145-FEDER-000004), Project UID/ QUI/50006/2019 - REQUIMTE-LAQV and strategic project PEst-OE/ AGR/UI0690/2014 - CIMO, all funded by FCT/MCTES through national funds. Ítala G. Marx also acknowledges the Ph.D. research grant (SFRH/BD/137283/2018) provided by FCT.

\section{References}

[1] C. Villatoroa, L. Vera, H. Gygax, Comparative study of odours present in twin fragrances by GC-sniffing-ToFMS, Chem. Eng. Trans. 54 (2016) 133-138 https://doi. org/10.3303/CET1654023.
[2] A. van Asten, The importance o GC and GC-MS in perfume analysis, Trends Anal. Chem. 21 (2002) 698-708 https://doi.org/10.1016/S0165-9936(02)00807-5.

[3] A. Cisvert, M. López-Nogueroles, A. Salvador, Essential oils: analytical methods to control the quality of perfumes, in: K. Ramawat, J.M. Mérillon (Eds.), Natural Products, Springer, Berlin, Heidelberg, 2013, pp. 3287-3310.

[4] N. Nibbe, H. Gygax, B. Maxeiner, Odour measurement for improved scent performance in consumer goods, SOFW J. (Seifen Ole Fette Wachse) 141 (2015) 42-46.

[5] J. Gebicki, B. Szulczynski, M. Kaminski, Determination of authenticity of brand perfume using electronic nose prototypes, Meas. Sci. Technol. 26 (2015) 125103https://doi.org/10.1088/0957-0233/26/12/125103.

[6] T. Nakamoto, A. Fukuda, T. Moriizumi, Perfume and flavor identification by odor sensing system using quartz-resonator sensor array and neural-network pattern recognition, Transducers 91 (1991) 355-358.

[7] M. Cano, V. Borrego, J. Roales, J. Idígoras, T. Lopes-Costa, P. Mendoza, J.M. Pedrosa, Rapid discrimination and counterfeit detection of perfumes by an electronic olfactory system, Sens. Actuators B Chem. 156 (2011) 319-324 https:// doi.org/10.1016/j.snb.2011.04.039.

[8] R. Singh, An intelligent system for odour discrimination, Proceedings - 1st IEEE International Workshop on Electronic Design, Test and Applications DELTA, 2002, pp. 489-491 art. no. 994681 https://doi.org/10.1109/DELTA.2002.994681.

[9] B. Bahraminejad, S. Basri, M. Isa, Z. Hambali, Application of a sensor array based on capillary-attached conductive gas sensors for odor identification, Meas. Sci. Technol. 21 (2010) 085204https://doi.org/10.1088/0957-0233/21/8/085204.

[10] A. Branca, P. Simonian, M. Ferrante, E. Novas, R.M. Negri, Electronic nose based discrimination of a perfumery compound in a fragrance, Sens. Actuators B Chem. 92 (2003) 222-227 https://doi.org/10.1016/S0925-4005(03)00270-3.

[11] B. Karlik, K. Yuksek, Fuzzy clustering neural networks for real-time odor recognition system, J. Autom. Methods Manage. Chem. 2007 (2007) 38405https://doi.org/ $10.1155 / 2007 / 38405$.

[12] T. Temel, B. Karlik, An improved odor recognition system using learning vecto quantization with a new linear discriminant analysis, Neural Netw. World 17 (2007) 287-294.

[13] W. Jatmiko, T. Fukuda, F. Arai, B. Kusumoputro, Artificial odor discrimination system using multiple qartz resonator sensors and various neural networks for recognizing fragrance mixtures, IEEE Sens. J. 6 (2006) 223-233 https://doi.org/10. 1109/JSEN.2005.858435.

[14] E. Esme, B. Karlikb, Fuzzy c-means based support vector machines classifier for perfume recognition, Appl. Soft Comput. J. 46 (2016) 452-458 https://doi.org/10. 1016/j.asoc.2016.05.030.

[15] T. Ye, C. Jin, J. Zhou, X. Li, H. Wang, P. Deng, Y. Yang, Y. Wu, X. Xiao, Can odors of TCM be captured by electronic nose? The novel quality control method for musk by electronic nose coupled with chemometrics, J. Pharm. Biomed. Anal. 55 (2011) 1239-1244 https://doi.org/10.1016/j.jpba.2011.03.018.

[16] T. Takeuchi, J. Montenegro, A. Hennig, M. Stefan, Pattern generation with synthetic sensing systems in lipid bilayer membranes, Chem. Sci. 2 (2011) 303-307 https:// doi.org/10.1039/c0sc00386g.

[17] M. Penza, G. Cassano, F. Tortorella, G. Zaccaria, Classification of food, beverages and perfumes by WO3 thin-film sensors array and pattern recognition techniques, Sens. Actuators B Chem. 73 (2001) 76-87 https://doi.org/10.1016/S09254005(00)00687-0.

[18] W. Guo, F. Gan, H. Kong, J. Wu, Signal model of electronic noses with metal oxide semiconductor, Chemometr. Intell. Lab. Syst. 143 (2015) 130-135 https://doi.org/ 10.1016/j.chemolab.2015.02.021.

[19] J. Poprawski, P. Boilot, F. Tetelin, Counterfeiting and quantification using an electronic nose in the perfumed cleaner industry, Sens. Actuators B Chem. 116 (2006) 156-160 https://doi.org/10.1016/j.snb.2005.11.074.

[20] M. Cole, J.A. Covington, J.W. Gardner, Combined electronic nose and tongue for a flavour sensing system, Sens. Actuators B Chem. 156 (2011) 832-839 https://doi. org/10.1016/j.snb.2011.02.049.

[21] P. Rattanawarinchai, P. Krongkrachang, T. Chodjarusawad, D. Phromyothin, Electrochemical sensor: preparation technique based on electronic tongue in fragrance, Mater. Today: Proceedings 4 (2017) 6410-6414 https://doi.org/10.1016/j. matpr.2017.06.146.

[22] A.C.A. Veloso, L.G. Dias, N. Rodrigues, J.A. Pereira, A.M. Peres, Sensory intensity assessment of olive oils using an electronic tongue, Talanta 146 (2016) 585-593 https://doi.org/10.1016/j.talanta.2015.08.071.

[23] L.G. Dias, N. Rodrigues, A.C.A. Veloso, J.A. Pereira, A.M. Peres, Monovarietal extravirgin olive oil classification: a fusion of human sensory attributes and an electronic tongue, Eur. Food Res. Technol. 242 (2016) 259-270 https://doi.org/10.1007/ s00217-015-2537-4.

[24] Í. Marx, N. Rodrigues, L.G. Dias, A.C.A. Veloso, J.A. Pereira, D.A. Drunkler, A.M. Peres, Sensory classification of table olives using an electronic tongue: analysis of aqueous pastes and brines, Talanta 162 (2017) 98-106 https://doi.org/10.1016/ j.talanta.2016.10.028.

[25] Í.M.G. Marx, N. Rodrigues, L.G. Dias, A.C.A. Veloso, J.A. Pereira, D.A. Drunkler, A.M. Peres, Quantification of table olives' acid, bitter and salty tastes using potentiometric electronic tongue fingerprints, LWT - Food Sci. Technol. (LebensmittelWissenschaft -Technol.) 79 (2017) 394-401 https://doi.org/10.1016/j.lwt.2017. 01.060 .

[26] S. Slim, N. Rodrigues, L.G. Dias, A.C.A. Veloso, J.A. Pereira, S. Oueslati, A.M. Peres, Application of an electronic tongue for Tunisian olive oils' classification according to olive cultivar or physicochemical parameters, Eur. Food Res. Technol. 243 (2017) 1459-1470 https://doi.org/10.1007/s00217-017-2856-8.

[27] A.C.A. Veloso, L.M. Silva, N. Rodrigues, L.P.G. Rebello, L.G. Dias, J.A. Pereira, A.M. Peres, Perception of olive oils sensory defects using a potentiometric taste device, Talanta 176 (2018) 610-618 https://doi.org/10.1016/j.talanta.2017.08. 
066.

[28] U. Harzalli, N. Rodrigues, A.C.A. Veloso, L.G. Dias, J.A. Pereira, S. Oueslati, A.M. Peres, A taste sensor device for unmasking admixing of rancid or winey-vinegary olive oil to extra virgin olive oil, Comput. Elelctron. Agric. 144 (2018) 222-231 https://doi.org/10.1016/j.compag.2017.12.016.

[29] Y. Kobayashi, M. Habara, H. Ikezazki, R. Chen, Y. Naito, K. Toko, Advanced taste sensors based on artificial lipids with global selectivity to basic taste qualities and high correlation to sensory scores, Sensors 10 (2010) 3411-3443 https://doi.org/ $10.3390 /$ s100403411.

[30] C.L. Gomes, A.C.A. Lima, M.C.L. Cândido, A.B.R. Silva, A.R. Loiola, R.F. Nascimento, Multivariate analysis of perfumes by ultraviolet spectrophotometry, J. Braz. Chem. Soc. 26 (2015) 1730-1736 https://doi.org/10.5935/ 0103-5053.20150135.

[31] N. Rodrigues, Í.M.G. Marx, S. Casal, L.G. Dias, A.C.A. Veloso, J.A. Pereira, A.M. Peres, Application of an electronic tongue as a single-run tool for olive oils' physicochemical and sensory simultaneous, Assessment 197 (2019) 363-373 https://doi.org/10.1016/j.talanta.2019.01.055.

[32] C.M. Bishop, Pattern Recognition and Machine Learning, first ed., Springer, New
York, 2006.

[33] J. Cadima, J.O. Cerdeira, M. Minhoto, Computational aspects of algorithms for variable selection in the context of principal components, Comput. Stat. Data Anal 47 (2004) 225-236 https://doi.org/10.1016/j.csda.2003.11.001.

[34] J. Cadima, J.O. Cerdeira, P.D. Silva, M. Minhoto, The subselect R package, Accessed date: http://cran.rproject.org/web/packages/subselect/vignettes/subselect.pdf/, (2012), Accessed date: 15 February 2016.

[35] W.N. Venables, B.D. Ripley, Modern Applied Statistics with S (Statistics and Computing), fourth ed., Springer, New York, 2002.

[36] R. Prata, J.A. Pereira, N. Rodrigues, L.G. Dias, A.C.A. Veloso, S. Casal, A.M. Peres, Olive oil total phenolic contents and sensory sensations trends during oven and microwave heating processes and their discrimination using an electronic tongue, $\mathrm{J}$. Food Qual. (2018) 78264282018 https://doi.org/10.1155/2018/7826428.

[37] L.G. Dias, A.M. Peres, A.C.A. Veloso, F.S. Reis, M. Vilas Boas, A.A.S.C. Machado, An electronic tongue taste evaluation: identification goat milk adulterations with bovine milk, Sens. Actuators B Chem. 136 (2009) 209-217 https://doi.org/10.1016/j. snb.2008.09.025. 